\title{
Solving the Navier Stokes Flow Equations of Micro-Polar Fluids by Adomian Decomposition Method
}

\author{
Villevo Adanhounmè ${ }^{1}$, François de Paule Codo ${ }^{2}$, Alain Adomou ${ }^{3}$ \\ ${ }^{1}$ International Chair of Mathematical Physics and Applications, University of Abomey-Calavi, \\ 072 B.P. 50 Cotonou, Republic of Benin \\ ${ }^{2}$ Laboratory for Applied Mechanics and Energetic (LEMA), Ecole Polytechnique d'Abomey-Calavi, \\ University of Abomey Calavi., 01 B.P.2009 Cotonou, Republic of Benin \\ ${ }^{3}$ Technological Institute of Lokossa. (IUT Lokossa), University of Abomey- Calavi, \\ B.P. 133 Lokossa, Republic of Benin
}

Keywords: Navier Stokes flow, change of variable, slip boundary conditions, micro-polar fluid

\begin{abstract}
In this paper we investigate the Navier Stokes flow equations of micro-polar fluids by peristaltic pumping through the cylindrical tube. Taking into account the slip boundary conditions at the wall and using the suitable change of variables, we transform these equations into the ordinary differential equations for which we apply the Adomian decomposition method. Doing so we obtain the stream function, the axial velocity, the micro-polar vector and the pressure.
\end{abstract}

\section{Introduction}

The fluid mechanics studies the behavior of particles at every point within the domain under various physical conditions. For describing the physical phenomena in fluid mechanics one uses the mathematical model of motion such as Navier-Stokes equations. It is a well known fact that a few exact solutions of the Navier-Stokes equations are known even now. This has been largely due to the complexity of the system of differential equations. In the absence of a general solution it is often convenient to experiment with models to obtain information on the flow phenomena e.g. the velocity distribution, flow pattern, pressure losses, etc. A special case of fluids, namely the micropolar fluids was first introduced in [1]. In a series of remarquable papers [2] to [12] the peristaltic transport of incompressible fluids and the Stokes flow of non-Newtonian fluids through different geometries of the flow pattern by peristaltic pumping have been studied. In [5], the micro-polar fluids represent the fluids consisting of rigid randomly oriented (spherical) particles suspended in a viscous medium, where the deformation of the particles is ignored. As physical phenomenon, peristaltic pumping is the pumping of the fluid induced due to the progressive waves of contraction or expansion and traveling along the walls of the vessel containing the fluid.

In fluid mechanics, most of problems are nonlinear. It is very important to develop efficient methods to solve them. One of the methods is the Adomian decomposition method(ADM) for solving a wide range of physical problems. This method is one of the semi-exact methods which does not need linearization or discretization. Several modifications were improved its ability in [13] to [18]. An advantage of this method is that, it can provide analytical approximation or an approximated solution to a wide class of nonlinear equations without linearization, pertubationclosure approximation or discretization methods. ADM abilities attracted many authors to use this method for solving fluid dynamics problems.

Applying the long wavelength assumption (the wave number too small with respect to 1) and the low Reynolds number approximation, the Stokes flow was studied in [5]. In this paper we investigate the analytical solutions to Navier-Stokes equations of micro-polar fluids by peristaltic pumping through the cylindrical tube, taking into account the slip boundary conditions at the wall without a priori assumptions on the flow equations. The rest of this paper is organized as follows: in the next section we present the details of the models we analyze. In the section 3 we use the Adomian decomposition method for solving the Navier-Stokes equations. In the conclusion, we summarize our results. 


\section{Mathematical Model}

Consider the reference fixed frame $\left(r^{\prime}, \theta^{\prime}, x^{\prime}\right)$ and the reference wave frame $(r, \theta, x)$. Then, the transformations from the .xed frame to the wave frame are given by

$$
r=r^{\prime}, x=x^{\prime}-c t, v=v^{\prime}, u=u^{\prime}-c
$$

where $\left(v^{\prime}, u^{\prime}\right)$ and $(v, u)$ are the radial and axial velocity components in the reference fixed frame and the reference wave frame, respectively, and $\mathrm{c}$ is the wave velocity.

The governing equations of the flow of an incompressible micro-polar fluid in the absence of body force and body couple in the reference wave frame [5]

$$
\begin{gathered}
\nabla . V=0, \\
p(V, \nabla V)=-\nabla p+\kappa \nabla \times W+(\mu+\kappa) \nabla^{2} V, \\
p j(V . \nabla W)=-2 \kappa W+\kappa \nabla \times V-\gamma(\nabla \times \nabla \times W)+(\alpha+\beta+\gamma) \nabla(\nabla . W),
\end{gathered}
$$

where $V=(v, 0, u)$ and $W=(0, \omega, 0)$ are the velocity vector and the micro-rotation vector, respectively, is the fluid pressure, $\rho$ is the fluid density and $\mathrm{j}$ is the micro-gyration parameter. The constants $\mu, \kappa, \alpha, \beta$ and $\gamma$ are material constants satisfying

$2 \mu+\kappa \geq 0$,

$3 \alpha+\beta+\gamma \geq 0, \alpha \geq|\beta|$

The equation of wall motion in the reference wave frame is

$$
\mathrm{H}=\alpha+\beta \sin \frac{2 \pi x}{\lambda}
$$

We non-dimensionnalize the different variables as follows:

$$
\left\{\begin{array}{c}
\bar{r}=\frac{r}{a}, \bar{x}=\frac{x}{\lambda}, \bar{u}=\frac{u}{c}, \bar{v}=\frac{\lambda}{c a} v \\
\bar{\omega}=\frac{a}{c} \omega, \bar{p}=\frac{a^{2}}{\lambda c \mu} p \\
\bar{i}=\frac{c}{\lambda} t, h=\frac{H}{a}, \bar{j}=\frac{j}{a^{2}}
\end{array}\right.
$$

Using (7) in (2) to (4) and (6) and dropping the bar, we get

$$
\begin{aligned}
& \frac{\partial v}{\partial r}+\frac{v}{r}+\frac{\partial u}{\partial x}=0, \\
& \operatorname{Re} \delta^{3}\left(v \frac{\partial v}{\partial r}+u \frac{\partial v}{\partial x}\right)=-\frac{\partial p}{\partial r}+\frac{\delta^{2}}{1-N}\left(-N \frac{\partial \omega}{\partial x}+\frac{\partial^{2} v}{\partial r^{2}}+\frac{\partial v}{r \partial r}-\frac{v}{r^{2}}+\delta^{2} \frac{\partial^{2} r}{\partial x^{2}}\right) \\
& \operatorname{Re} \delta\left(v \frac{\partial u}{\partial r}+u \frac{\partial u}{\partial x}\right)=-\frac{\partial p}{\partial x}+\frac{1}{1-N}\left(\frac{N}{r} \frac{\partial(r \omega)}{\partial r}+\frac{\partial^{2} u}{\partial r^{2}}+\frac{\partial u}{r \partial r}+\delta^{2} \frac{\partial^{2} u}{\partial x^{2}}\right) \\
& j R e \frac{\delta(1-N)}{N}\left(v \frac{\partial \omega}{\partial r}+u \frac{\partial \omega}{\partial x}\right)=-2 \omega+\left(\delta^{2} \frac{\partial v}{\partial x}-\frac{\partial u}{\partial r}\right)+\frac{2-N}{m^{2}}\left(\frac{\partial}{\partial r}\left(\frac{1}{r} \frac{\partial(r \omega)}{\partial r}\right)+\delta^{2} \frac{\partial^{2} \omega}{\partial x^{2}}\right) \\
& h(x)=1+\phi \sin (2 \pi x)
\end{aligned}
$$


where $\delta=\frac{a}{\lambda}$ is the wave number, $\phi=\frac{b}{a}$ is the amplitude, $R e=\frac{\rho a c}{\mu}$ is the Reynolds number, $m=\sqrt{a^{2} \kappa(2 \mu+\kappa) / \gamma(\mu+\kappa)}$

Taking into account the components of the velocity through the stream function $\psi$, i.e.

$v=-\frac{1}{r} \frac{\partial \psi}{\partial x}, u=-\frac{1}{r} \frac{\partial \psi}{\partial r}$

we can write (9) to (11) in the form

$\operatorname{Re} \delta^{3}\left(-\frac{1}{r^{3}}\left(\frac{\partial \psi}{\partial x}\right)^{2}+\frac{1}{r^{2}} \frac{\partial \psi}{\partial x} \frac{\partial^{2} \psi}{\partial x \partial r}-\frac{1}{r^{2}} \frac{\partial \psi}{\partial r} \frac{\partial^{2} \psi}{\partial x^{2}}\right)+\frac{\delta^{2} N}{r(1-N)} \frac{\partial(r \omega)}{\partial x}=$

$-\frac{\partial p}{\partial r}+\frac{\delta^{2}}{(1-N)}\left(\frac{1}{r^{2}} \frac{\partial^{2} \psi}{\partial x \partial r}-\frac{1}{r} \frac{\partial^{3} \psi}{\partial r^{2} \partial x}-\frac{\delta^{2}}{r} \frac{\partial^{3} \psi}{\partial x^{3}}\right)$

$\operatorname{Re} \delta\left(\frac{1}{r^{3}} \frac{\partial \psi}{\partial x} \frac{\partial \psi}{\partial r}-\frac{1}{r^{2}} \frac{\partial \psi}{\partial x} \frac{\partial^{2} \psi}{\partial r^{2}}+\frac{1}{r^{2}} \frac{\partial \psi}{\partial r} \frac{\partial^{2} \psi}{\partial x \partial r}\right)-\frac{N}{r(1-N)} \frac{\partial(r \omega)}{\partial r}=$

$-\frac{\partial p}{\partial x}+\frac{1}{(1-N)}\left(\frac{1}{r^{3}} \frac{\partial \psi}{\partial r}-\frac{1}{r^{2}} \frac{\partial^{2} \psi}{\partial r^{2}}+\frac{1}{r} \frac{\partial^{3} \psi}{\partial r^{3}}+\frac{\delta^{2}}{r} \frac{\partial^{3} \psi}{\partial x^{2} \partial r}\right)$

$j R e \delta \frac{1-N}{N}\left(-\frac{1}{r} \frac{\partial \psi}{\partial x} \frac{\partial \omega}{\partial r}+\frac{1}{r} \frac{\partial \psi}{\partial r} \frac{\partial \omega}{\partial x}\right)=-2 \omega+\left(-\frac{\delta^{2}}{r} \frac{\partial^{2} \psi}{\partial x^{2}}-\frac{1}{r} \frac{\partial^{2} \psi}{\partial r^{2}}+\frac{1}{r^{2}} \frac{\partial \psi}{\partial r}\right)$

$+\frac{2-N}{m^{2}}\left(\frac{\partial}{\partial r}\left(\frac{\partial(r \omega)}{r \partial r}\right)+\delta^{2} \frac{\partial^{2} \omega}{\partial x^{2}}\right)$

The corresponding boundary conditions in the wave frame are as follows:

1. The regularity condition is

$$
\frac{\partial u}{\partial r}=0 \quad \text { at } r=0
$$

2. The slip boundary condition is

$$
\begin{array}{ll}
u=-1-k_{n} \frac{\partial u}{\partial r} & \text { at } r=h \\
\omega=0 & \text { at } r=h \\
u=0, \omega=0 & \text { at } r=0
\end{array}
$$

where $_{k n}=L / a$ is the dimensionless slip parameter and $\mathrm{L}$ is the dimensional slip parameter.

\section{Analytical Solutions}

In this section we provide the analytical solutions to these equations using the Adomian decomposition technique.

Setting

$$
\begin{aligned}
& \psi(r, x)=f(\eta) \\
& \omega(r, x)=\frac{1}{r} g(\eta) \\
& p(r, x)=q(\eta) \\
& \eta=r-\delta^{2} x
\end{aligned}
$$


Where $f, g, q$ are continuously differentiable functions, we transform (14) to (20) as

$$
\begin{aligned}
& q^{\prime}(\eta)=\frac{-1}{r^{2}(1-N)\left(1+\delta^{-2}\right)} f^{\prime}(\eta) \\
& g^{\prime}(\eta)=\frac{-1}{N}\left(\frac{\operatorname{Re}(1-N)}{r^{2} \delta} f^{\prime 2}(\eta)+\frac{1}{r^{2}\left(1+\delta^{-2}\right)} f^{\prime}(\eta)-\frac{1}{r} f^{\prime \prime}(\eta)+\left(1+\delta^{-2}\right) f^{\prime \prime \prime}(\eta)\right) \\
& \frac{r(2-N)}{m^{2}}\left(1+\delta^{-2}\right) g^{\prime \prime}(\eta)-\frac{2-N}{m^{2}} g^{\prime}(\eta)-2 r g(\eta)=r\left(1+\delta^{-2}\right) f^{\prime \prime}(\eta)-f^{\prime}(\eta) \\
& f^{\prime}\left(-\delta^{2} x\right)=f^{\prime \prime}\left(-\delta^{2} x\right)=0, \lim _{\mathrm{r} \rightarrow 0} \frac{1}{r} g(\eta)=0, g(\bar{\eta})=0 \\
& k_{n} h f^{\prime \prime}(\bar{\eta})+\left(h-k_{n}\right) f^{\prime}(\bar{\eta})=-h^{2}, \quad \bar{\eta}=h-\delta^{2} x
\end{aligned}
$$

where (.)' stands for $\frac{d(.)}{d \eta}$

The general solution to (27) is

$$
\begin{aligned}
& g(\eta)=e^{\lambda_{1} \eta}\left\{K_{1}-\frac{1}{\sqrt{\Delta}} \int_{0}^{\eta}\left[\left(1+\delta^{-2}\right) r f^{\prime \prime}(\xi)-f^{\prime}(\xi)\right] e^{\lambda_{1} \xi} \mathrm{d} \xi\right\} \\
& +e^{\lambda_{2} \eta}\left\{K_{2}+\frac{1}{\sqrt{\Delta}} \int_{0}^{\eta}\left[r\left(1+\delta^{-2}\right) f^{\prime \prime}(\xi)-f^{\prime}(\xi)\right] e^{\lambda_{2} \xi} \mathrm{d} \xi\right\}
\end{aligned}
$$

Where

$$
\begin{aligned}
& \Delta=\left(\frac{2-N}{m^{2}}\right)^{2}+\frac{\delta r^{2}}{m^{2}}(2-N)\left(1+\delta^{-2}\right) \\
& K_{1}=K_{1}(x), K_{2}=K_{2}(x) \\
& \lambda_{1}=\frac{m^{2}}{2 r(2-N)\left(1+\delta^{-2}\right)}\left(\frac{2-N}{m^{2}}-\sqrt{\Delta}\right) \\
& \lambda_{2}=\frac{m^{2}}{2 r(2-N)\left(1+\delta^{-2}\right)}\left(\frac{2-N}{m^{2}}-\sqrt{\Delta}\right)
\end{aligned}
$$

Using (28) and $f(0)=0$ in (30) we obtain

$$
\begin{aligned}
& g(\eta)=e^{\lambda_{1} \eta}\left\{K_{1}-\frac{1}{\sqrt{\Delta}} \int_{0}^{\eta}\left[\left(1+\delta^{-2}\right) r \lambda_{1}-1\right] f^{\prime}(\xi) e^{-\lambda_{1} \xi} d \xi\right\} \\
& +e^{\lambda_{2} \eta}\left\{K_{2}+\frac{1}{\sqrt{\Delta}} \int_{0}^{\eta}\left[r \lambda_{2}\left(1+\delta^{-2}\right)-1\right] f^{\prime}(\xi) e^{-\lambda_{2} \xi} d \xi\right\} \\
& K_{1}=\frac{-m^{2}}{2-N} f\left(-\delta^{-2} x\right) \\
& K_{2}=-K_{1} e^{\left(\bar{\lambda}_{1}-\bar{\lambda}_{2}\right) \eta}+\frac{1}{\sqrt{\Delta}} \int_{0}^{\eta}\left\{\left[\left(1+\delta^{-2}\right) h \bar{\lambda}_{1}-1\right] e^{\left(\bar{\lambda}_{1}-\bar{\lambda}_{2}\right) \eta-\bar{\lambda}_{1} \xi}-\left[\left(1+\delta^{-2}\right) h \bar{\lambda}_{2}-1\right] e^{\bar{\lambda}_{2} \xi}\right\} f^{\prime}(\xi) d \xi \\
& \bar{\Delta}=\left(\frac{2-N}{m^{2}}\right)^{2}+\frac{\delta h^{2}}{m^{2}}(2-N)\left(1+\delta^{-2}\right) \\
& \bar{\lambda}_{1}=\frac{m^{2}}{2 h(2-N)\left(1+\delta^{-2}\right)}\left(\frac{2-N}{m^{2}}-\sqrt{\bar{\Delta}}\right) \\
& \bar{\lambda}_{2}=\frac{m^{2}}{2 h(2-N)\left(1+\delta^{-2}\right)}\left(\frac{2-N}{m^{2}}+\sqrt{\bar{\Delta}}\right)
\end{aligned}
$$


Let us transform (26) and (35) into the nonlinear integral equation. Integrating from 0 to $\eta$ the equality of (26) and the first derivative of (35), we obtain the following equation

$$
\begin{aligned}
& f^{\prime \prime}(\eta)-\left\{\frac{1}{r\left(1+\delta^{-2}\right)} f^{\prime}(\eta)-\int_{0}^{\eta}\left[\frac{\operatorname{Re}(1-N)}{r^{2} \delta\left(1+\delta^{-2}\right)} f^{\prime 2}(z)+\left(\frac{r N}{\sqrt{\Delta}}\left(\lambda_{2}-\lambda_{1}\right)+\frac{1}{r^{2}\left(1+\delta^{-2}\right)^{2}} f^{\prime}(z)\right)\right] d z\right. \\
& \left.-\frac{N}{\left(1+\delta^{-2}\right) \sqrt{\Delta}} \int_{0}^{\eta} \int_{0}^{z}\left[\lambda_{1}\left(1-\lambda_{1} r\left(1+\delta^{-2}\right)\right) e^{\lambda_{1}(z-s)}+\lambda_{2}\left(\lambda_{2} r\left(1+\delta^{-2}\right)-1\right) e^{\lambda_{2}(z-s)}\right] f^{\prime}(s) d s d z\right\} \\
& =-\frac{N}{1+\delta^{-2}} \int_{0}^{\eta}\left(\lambda_{1} K_{1} e^{\lambda_{1} z}+\lambda_{2} K_{2} e^{\lambda_{2} z}\right) d z,
\end{aligned}
$$

Integrating from 0 to $\eta(40)$ and using (29) in the obtained result we can write

$$
\begin{aligned}
& \varphi(\eta)-\left\{\frac{1}{r\left(1+\delta^{-2}\right)} \int_{0}^{\eta} \varphi(z) d z-\int_{0}^{\eta} \int_{0}^{\xi}\left[\frac{\operatorname{Re}(1-N)}{r^{2} \delta\left(1+\delta^{-2}\right)} \varphi^{2}(z)+\left(\frac{r N}{\sqrt{\Delta}}\left(\lambda_{2}-\lambda_{1}\right)+\frac{1}{r^{2}\left(1+\delta^{-2}\right)^{2}}\right) \varphi(z)\right] d z d \xi\right. \\
& \left.-\frac{N}{\left(1+\delta^{-2}\right) \sqrt{\Delta}} \int_{0}^{\eta} \int_{0}^{\xi} \int_{0}^{z}\left[\lambda_{1}\left(1-\lambda_{1} r\left(1+\delta^{-2}\right)\right) e^{\lambda_{1}(z-s)}+\lambda_{2}\left(\lambda_{2} r\left(1+\delta^{-2}\right)-1\right) e^{\lambda_{2}(z-s)}\right] \varphi(s) d s d z d \xi\right\} \\
& =-\frac{N}{1+\delta^{-2}} \int_{0}^{\eta} \int_{0}^{\xi}\left(\lambda_{1} K_{1} e^{\lambda_{1} z}+\lambda_{2} K_{2} e^{\lambda_{2} z}\right) d z d \xi+K_{3}(x),
\end{aligned}
$$

which is a nonlinear functional equation such as the following

where

$$
\varphi(\eta)-G(\varphi)=F(\eta)+K_{3}(x)
$$

$$
\begin{aligned}
& K_{3}(x)=\frac{N}{1+\delta^{-2}} \int_{0}^{\eta} \int_{0}^{\xi}\left(\bar{\lambda}_{1} K_{1} e^{\bar{\lambda}_{1} z}+\bar{\lambda}_{2} K_{2} e^{\bar{\lambda}_{2} z}\right) d z d \xi+\frac{1}{k_{n}-h}\left(h^{2}+k_{n} h g f^{\prime \prime}(\bar{\eta})\right) \\
& -\left\{\frac{1}{h\left(1+\delta^{-2}\right)} \int_{0}^{\eta} f^{\prime}(z) d z-\int_{0}^{\eta} \int_{0}^{\xi}\left[\frac{R e(1-N)}{\left(h^{2} \delta\right)\left(1+\delta^{-2}\right)} f^{\prime 2}(z)+\left(\frac{h N}{\sqrt{\Delta}}\left(\bar{\lambda}_{2}-\bar{\lambda}_{1}\right)\right.\right.\right. \\
& \left.\left.+\frac{1}{h^{2}\left(1+\delta^{-2}\right)^{2}}\right) f^{\prime}(z)\right] d z d \xi-\frac{N}{\left(1+\delta^{-2}\right) \sqrt{\bar{\Delta}}} \int_{0}^{\eta} \int_{0}^{\xi} \int_{0}^{z}\left[\bar{\lambda}_{1}\left(1-\overline{\lambda_{1}} h\left(1+\delta^{-2}\right)\right) e^{\bar{\lambda}_{1}(z-s)}\right. \\
& \left.\left.+\bar{\lambda}_{2}\left(\bar{\lambda}_{2} h\left(1+\delta^{-2}\right)-1\right) e^{\bar{\lambda}_{2}(z-s)}\right] f^{\prime}(s) d s d z d \xi\right\}, \\
& \varphi(\eta)=f^{\prime}(\eta) \\
& F(\eta)=\frac{N}{1+\delta^{-2}}\left[K_{1}\left(\frac{1}{\lambda_{1}}+\eta-\frac{1}{\lambda_{1}} e^{\lambda_{1} \eta}\right)+K_{2}\left(\frac{1}{\lambda_{2}}+\eta-\frac{1}{\lambda_{2}} e^{\lambda_{2} \eta}\right)\right] \\
& G(\varphi)=\frac{1}{r\left(1+\delta^{-2}\right)} \int_{0}^{\eta} \varphi(z) d z-\int_{0}^{\eta} \int_{0}^{\xi}\left[\frac{R e(1-N)}{r^{2}\left(1+\delta^{-2}\right)} \varphi^{2}(z)+\left(\frac{r N}{\sqrt{\Delta}}\left(\lambda_{2}-\lambda_{1}\right)+\frac{1}{r^{2}\left(1+\delta^{-2}\right)^{2}}\right) \varphi(z)\right] d z d \xi \\
& -\frac{N}{\left(1+\delta^{-2}\right) \sqrt{\Delta}} \int_{0}^{\eta} \int_{0}^{\xi} \int_{0}^{z}\left[\lambda_{1}\left(1-\lambda_{1} r\left(1+\delta^{-2}\right)\right) e^{\lambda_{1}(z-s)}+\lambda_{2}\left(\lambda_{2} r\left(1+\delta^{-2}\right)-1\right) e^{\lambda_{2}(z-s)}\right] \varphi(s) d s d z d \xi
\end{aligned}
$$

$\mathrm{G}$ is a nonlinear operator from a Hilbert space $H$ into $H$. In [13] and [14] one has developed a decomposition technique for solving nonlinear functional equation such as (42). Without loss of generality, we set $K_{3}(x)=0$ and we assume that (42) has a unique solution. The Adomian's technique allows us to find the solution of (42) as an infinite series $\varphi=\Sigma_{\mathrm{n} \geq 0}$ using the following scheme:

$$
\begin{aligned}
& \varphi_{0}=F(\eta)=\frac{N}{1+\delta^{-2}}\left[K_{1}\left(\frac{1}{\lambda_{1}}+\eta-\frac{1}{\lambda_{1}} e^{\lambda_{1} \eta}\right)+K_{2}\left(\frac{1}{\lambda_{2}}+\eta-\frac{1}{\lambda_{2}} e^{\lambda_{2} \eta}\right)\right] \\
& \varphi_{1}=A_{0}=G\left(\varphi_{0}\right) \\
& \varphi_{2}=A_{1}=\varphi_{1} G^{\prime}\left(\varphi_{0}\right)
\end{aligned}
$$


Bulletin of Mathematical Sciences and Applications Vol. 2

35

$$
\begin{aligned}
& \varphi_{3}=A_{2}=\varphi_{2} G^{\prime}\left(\varphi_{0}\right)+\frac{1}{2} \varphi_{1} \varphi_{1} G^{\prime \prime}\left(\varphi_{0}\right) \\
& \varphi_{4}=A_{3}=\varphi_{3} G^{\prime}\left(\varphi_{0}\right)+\frac{1}{2} \varphi_{1} \varphi_{2} G^{\prime \prime}\left(\varphi_{0}\right) \\
& \varphi_{n+1}=\frac{1}{n !}\left[\frac{d^{n}}{d \lambda^{n}} G\left[\sum_{i \geq 0} \lambda^{i} \varphi_{i}\right]\right]=0 \\
& =\varphi_{n} G^{\prime}\left(\varphi_{0}\right)+\frac{G^{\prime \prime}\left(\varphi_{0}\right)}{n} \sum_{i=0}^{n-1}(n-1) \varphi_{i} \varphi_{n-1}, n \geq 2,
\end{aligned}
$$

where $\mathrm{A}_{n}\left(\varphi_{0}, \varphi_{1}, \varphi_{2}, \ldots, \varphi_{n}\right)$ are polynomials of $\varphi_{0}, \varphi_{1}, \varphi_{2}, \ldots, \varphi_{n}$ and

$$
\begin{aligned}
& G\left(\varphi_{0}\right)=\frac{N}{r\left(1+\delta^{-2}\right)^{2}}\left[K_{1}\left(\frac{1}{\lambda_{1}^{2}}+\frac{1}{\lambda_{1}} \eta+\frac{1}{2} \eta^{2}-\frac{1}{\lambda_{1}^{2}} e^{\lambda_{1} \eta}\right)+K_{2}\left(\frac{1}{\lambda_{2}^{2}}+\frac{1}{\lambda_{2}} \eta+\frac{1}{2} \eta^{2}-\frac{1}{\lambda_{2}^{2}} e^{\lambda_{2} \eta}\right)\right] \\
& -\frac{\operatorname{Re}(1-N) N^{2}}{r^{2} \delta\left(1+\delta^{-2}\right)^{3}}\left\{K_{1}^{2}\left[\frac{-7}{3 \lambda_{1}^{4}}-\frac{5}{6 \lambda_{1}^{3}} \eta+\frac{1}{12}\left(\frac{1}{\lambda_{1}}+\eta\right)^{4}+\frac{2}{\lambda_{1}^{4}}\left(-\lambda_{1} \eta+1\right) e^{\lambda_{1} \eta}+\frac{1}{4 \lambda_{1}^{4}} e^{2 \lambda_{1} \eta}\right]\right. \\
& +2 K_{1} K_{2}\left[\frac{1}{\lambda_{1} \lambda_{2}^{3}}+\frac{1}{\lambda_{2} \lambda_{1}^{3}}-\frac{2}{\lambda_{1}^{4}}-\frac{2}{\lambda_{2}^{4}}+\frac{1}{\lambda_{1} \lambda_{2}\left(\lambda_{1}+\lambda_{2}\right)^{2}}+\left(\frac{1}{\lambda_{1} \lambda_{2}^{2}}+\frac{1}{\lambda_{2} \lambda_{1}^{2}}-\frac{1}{\lambda_{1}^{3}}-\frac{1}{\lambda_{2}^{3}}+\frac{1}{\lambda_{1} \lambda_{2}\left(\lambda_{1}+\lambda_{2}\right)}\right) \eta\right. \\
& +\frac{1}{2 \lambda_{1} \lambda_{2}} \eta^{2}+\frac{1}{6 \lambda_{1} \lambda_{2}}\left(\lambda_{1}+\lambda_{2}\right) \eta^{3}+\frac{1}{12} \eta^{4}+\left(-\frac{1}{\lambda_{1}^{3} \lambda_{2}}+\frac{1}{\lambda_{1}^{4}}-\frac{1}{\lambda_{1}^{3}} \eta\right) e^{\lambda_{1} \eta}+\left(-\frac{1}{\lambda_{2} \lambda_{2}^{3}}+\frac{1}{\lambda_{2}^{4}}-\frac{1}{\lambda_{2}^{3}} \eta\right) e^{\lambda_{2} \eta} \\
& \left.\left.-\frac{1}{\lambda_{1} \lambda_{2}\left(\lambda_{1}+\lambda_{2}\right)^{2}} e^{\left(\lambda_{1}+\lambda_{2}\right) \eta}\right]+K_{2}^{2}\left[\frac{-7}{3 \lambda_{2}^{4}}-\frac{5}{6 \lambda_{2}^{3}} \eta+\frac{1}{12}\left(\frac{1}{\lambda_{2}}+\eta\right)^{4}+\frac{2}{\lambda_{2}^{4}}\left(-\lambda_{2} \eta+1\right) e^{\lambda_{2} \eta}+\frac{1}{4 \lambda_{2}^{4}} e^{2 \lambda_{2} \eta}\right]\right\} \\
& -\frac{N}{1+\delta^{-2}}\left(\frac{r N}{\sqrt{\Delta}}\left(\lambda_{2}-\lambda_{1}\right)+\frac{1}{r^{2}\left(1+\delta^{-2}\right)^{2}}\right)\left[K_{1}\left(\frac{1}{\lambda_{1}^{3}}+\frac{1}{\lambda_{1}^{2}} \eta+\frac{1}{2 \lambda_{1}} \eta^{2}+\frac{1}{6} \eta^{3}-\frac{1}{\lambda_{1}^{3}} e^{\lambda_{1} \eta}\right)\right. \\
& \left.+K_{2}\left(\frac{1}{\lambda_{2}^{3}}+\frac{1}{\lambda_{2}^{2}} \eta+\frac{1}{2 \lambda_{2}} \eta^{2}+\frac{1}{6} \eta^{3}-\frac{1}{\lambda_{2}^{3}} e^{\lambda_{2} \eta}\right)\right]-\frac{N^{2} \lambda_{1}\left(1-\lambda_{1} r\left(1+\delta^{-2}\right)\right)}{\left(1+\delta^{-2}\right)^{2} \sqrt{\Delta}} \\
& \left\{K_{1}\left[-\frac{4}{\lambda_{1}^{4}}-\frac{3}{\lambda_{1}^{3}} \eta-\frac{1}{\lambda_{1}^{2}} \eta^{2}-\frac{1}{6 \lambda_{1}} \eta^{3}+\left(-\frac{1}{\lambda_{1}^{3}} \eta+\frac{4}{\lambda_{1}^{4}}\right) e^{\lambda_{1} \eta}\right]+K_{2}\left[-\frac{1}{\lambda_{1}^{4}}\left(1+\frac{\lambda_{1}}{\lambda_{2}}\right)-\frac{\lambda_{1}+\lambda_{2}}{\lambda_{1}^{2} \lambda_{2}^{3}}\right.\right. \\
& -\left(\frac{1}{\lambda_{1} \lambda_{2}^{2}}+\frac{1}{\lambda_{1}^{2} \lambda_{2}}+\frac{1}{\lambda_{1}^{3}}\right) \eta-\frac{1}{2}\left(\frac{1}{\lambda_{1} \lambda_{2}}+\frac{1}{\lambda_{1}^{2}}\right) \eta^{2}-\frac{1}{6 \lambda_{1}} \eta^{3}+\frac{1}{\lambda_{1}^{2}}\left(\frac{1}{\lambda_{1} \lambda_{2}}+\frac{1}{\lambda_{1}^{2}}+\frac{1}{\lambda_{2}\left(\lambda_{2}-\lambda_{1}\right)}\right) e^{\lambda_{1} \eta} \\
& \left.\left.-\frac{1}{\lambda_{2}^{3}\left(\lambda_{2}-\lambda_{1}\right)} e^{\lambda_{2} \eta}\right]\right\}-\frac{N^{2} \lambda_{2}\left(\lambda_{2} r\left(1+\delta^{-2}\right)-1\right)}{\left(1+\delta^{-2}\right)^{2} \sqrt{\Delta}}\left\{K _ { 1 } \left[-\frac{1}{\lambda_{2}^{4}}\left(1+\frac{\lambda_{2}}{\lambda_{1}}\right)-\frac{\lambda_{1}+\lambda_{2}}{\lambda_{1}^{3} \lambda_{2}^{2}}-\left(\frac{1}{\lambda_{1} \lambda_{2}^{2}}+\frac{1}{\lambda_{1}^{2} \lambda_{2}}+\frac{1}{\lambda_{2}^{3}}\right)\right.\right. \\
& \left.-\frac{1}{2}\left(\frac{1}{\lambda_{1} \lambda_{2}}+\frac{1}{\lambda_{2}^{2}}\right) \eta^{2}-\frac{1}{6 \lambda_{2}} \eta^{3}+\frac{1}{\lambda_{1}^{3}\left(\lambda_{2}-\lambda_{1}\right)} e^{\lambda_{1} \eta}+\left(\frac{1}{\lambda_{1} \lambda_{2}^{3}}+\frac{1}{\lambda_{2}^{4}}-\frac{1}{\lambda_{1} \lambda_{2}^{2}\left(\lambda_{2}-\lambda_{1}\right)}\right) e^{\lambda_{2} \eta}\right] \\
& \left.+K_{2}\left[\frac{-4}{\lambda_{2}^{4}}-\frac{3}{\lambda_{2}^{3}} \eta-\frac{1}{\lambda_{2}^{2}} \eta^{2}-\frac{1}{6 \lambda_{2}} \eta^{3}+\left(-\frac{1}{\lambda_{2}^{3}} \eta+\frac{4}{\lambda_{2}^{4}}\right) e^{\lambda_{2} \eta}\right]\right\} \text {, }
\end{aligned}
$$




$$
\begin{aligned}
& G^{\prime}\left(\varphi_{0}\right)=\frac{1}{r\left(1+\delta^{-2}\right)} \eta-2 \frac{\operatorname{Re}(1-N) N}{r^{2} \delta\left(1+\delta^{-2}\right)^{2}}\left[K_{1}\left(\frac{1}{\lambda_{1}^{3}}+\frac{1}{\lambda_{1}^{2}} \eta+\frac{1}{2 \lambda_{1}} \eta^{2}+\frac{1}{6} \eta^{3}-\frac{1}{\lambda_{1}^{3}} e^{\lambda_{1} \eta}\right)\right. \\
& \left.+K_{2}\left(\frac{1}{\lambda_{2}^{3}}+\frac{1}{\lambda_{2}^{2}} \eta+\frac{1}{2 \lambda_{2}} \eta^{2}+\frac{1}{6} \eta^{2}-\frac{1}{\lambda_{2}^{3}} e^{\lambda_{2} \eta}\right)\right]+\frac{1}{2}\left(\frac{N r}{\sqrt{J}}\left(\lambda_{2}-\lambda_{1}\right)+\frac{1}{r^{2}\left(1+\delta^{-2}\right)^{2}}\right) \eta^{2} \\
& +\frac{N}{\left(1+\delta^{-2}\right) \sqrt{J}}\left(1-\lambda_{1} r\left(1+\delta^{-2}\right)\right)\left(\frac{1}{\lambda_{1}^{2}}+\frac{1}{\lambda_{1}} \eta+\frac{1}{2} \eta^{2}-\frac{1}{\lambda_{1}^{2}} e^{\lambda_{1} \eta}\right) \\
& +\frac{N}{\left(1+\delta^{-2}\right) \sqrt{J}}\left(\lambda_{2} r\left(1+\delta^{-2}\right)-1\right)\left(\frac{1}{\lambda_{2}^{2}}+\frac{1}{\lambda_{2}} \eta+\frac{1}{2} \eta^{2}-\frac{1}{\lambda_{2}^{2}} e^{\lambda_{2} \eta}\right), \\
& G^{\prime \prime}\left(\varphi_{0}\right)=-\frac{R e(1-N)}{r^{2} \delta\left(1+\delta^{-2}\right)} \eta^{2}
\end{aligned}
$$

Then we obtain

$$
\begin{aligned}
& f^{\prime}(\eta)=\varphi_{0}+G\left(\varphi_{0}\right)+G^{\prime}\left(\varphi_{0}\right) G\left(\varphi_{0}\right)+G\left(\varphi_{0}\right) G^{\prime 2}\left(\varphi_{0}\right)+\frac{1}{2} G^{\prime \prime}\left(\varphi_{0}\right) G^{2}\left(\varphi_{0}\right) \\
& +G\left(\varphi_{0}\right) G^{\prime 3}\left(\varphi_{0}\right)+\frac{3}{2} G^{\prime \prime}\left(\varphi_{0}\right) G^{\prime}\left(\varphi_{0}\right) G^{2}\left(\varphi_{0}\right)+\cdots \\
& +\varphi_{n} G^{\prime}\left(\varphi_{0}\right)+\frac{G n\left(\varphi_{0}\right)}{m} \sum_{i=1}^{n-1}(n-i) \varphi_{i} \varphi_{n-i}+\cdots
\end{aligned}
$$

and the approximated solution is defined by

$$
\begin{aligned}
& f^{\prime}(\eta)=\varphi_{0}+\varphi_{1}+\varphi_{2}+\varphi_{3}+\varphi_{4} \\
& =\varphi_{0}+G\left(\varphi_{0}\right)+G\left(\varphi_{0}\right) G^{\prime}\left(\varphi_{0}\right)+G\left(\varphi_{0}\right) G^{\prime 2}\left(\varphi_{0}\right)+\frac{1}{2} G^{\prime \prime}\left(\varphi_{0}\right) G^{2}\left(\varphi_{0}\right) \\
& +G\left(\varphi_{0}\right) G^{\prime 3}\left(\varphi_{0}\right)+\frac{3}{2} G^{\prime \prime}\left(\varphi_{0}\right) G^{\prime}\left(\varphi_{0}\right) G^{2}\left(\varphi_{0}\right) .
\end{aligned}
$$

Using (57) in (25) and (35), we arrive at the following results

Lemma. The stream function $\psi$ the axial velocity $u$, the micro-polar vector and the pressure $p$ are defined by

$$
\begin{aligned}
& \psi(r, x)=\int_{0}^{\eta}\left\{\varphi_{0}(\xi)+G\left[\varphi_{0}(\xi)\right]+G\left[\varphi_{0}(\xi)\right] G^{\prime}\left[\varphi_{0}(\xi)\right]+G\left[\varphi_{0}(\xi)\right] G^{n}\left[\varphi_{0}(\xi)\right]\right. \\
& \left.+\frac{1}{2} G^{\prime \prime}\left[\varphi_{0}(\xi)\right] G^{2}\left[\varphi_{0}(\xi)\right]+G\left[\varphi_{0}(\xi)\right] G^{\prime 3}\left[\varphi_{0}(\xi)\right]\right) \\
& \left.+\frac{3}{2} G^{\prime \prime}\left[\varphi_{0}(\xi)\right] G^{\prime}\left[\varphi_{0}(\xi)\right] G^{2}\left[\varphi_{0}(\xi)\right]\right\} d \xi . \\
& u(r, x)=\frac{1}{\gamma}\left\{\varphi_{0}(\eta)+G\left[\varphi_{0}(\eta)\right]+G\left[\varphi_{0}(\eta)\right] G^{\prime}\left[\varphi_{0}(\eta)\right]+G^{\prime 2}\left[\varphi_{0}(\eta)\right] G\left[\varphi_{0}(\eta)\right]\right. \\
& \left.+\frac{1}{2} G^{\prime \prime}\left[\varphi_{0}(\eta)\right] G^{2}\left[\varphi_{0}(\eta)\right]+G\left[\varphi_{0}(\eta)\right] G^{\prime 3}\left[\varphi_{0}(\eta)\right]+-\frac{3}{2} G^{\prime \prime}\left[\varphi_{0}(\eta)\right] G^{\prime}\left[\varphi_{0}(\eta)\right] G^{2}\left[\varphi_{0}(\eta)\right]\right\} \\
& \omega(r, x)=\frac{1}{r}\left\{K_{1} e^{\lambda_{1} \eta}+K_{2} e^{\lambda_{2} \eta}+\frac{1}{\sqrt{\Delta}} \int_{0}^{\eta}\left[\left[-\left(1+\delta^{-2}\right) \lambda_{1} r+1\right] e^{\lambda_{1}(\eta-\xi)}\right.\right. \\
& \left.+\left[\lambda_{2} r\left(1+\delta^{-2}\right)-1\right] e^{\lambda_{2}(\eta-\xi)}\right]\left[\varphi_{0}(\xi)+G\left[\varphi_{0}(\xi)\right]+G\left[\varphi_{0}(\xi)\right] G^{\prime}\left[\varphi_{0}(\xi)\right]\right. \\
& +G\left[\varphi_{0}(\xi)\right] G^{\prime 2}\left[\varphi_{0}(\xi)\right]+\frac{1}{2} G^{\prime \prime}\left[\varphi_{0}(\xi)\right] G^{2}\left[\varphi_{0}(\xi)\right]+G\left[\varphi_{0}(\xi)\right] G^{\prime 3}\left[\varphi_{0}(\xi)\right] \\
& \left.+\frac{a}{2} G^{\prime \prime}\left[\varphi_{0}(\xi)\right] G^{\prime}\left[\varphi_{0}(\xi)\right] G^{2}\left[\varphi_{0}(\xi)\right] d \xi\right] \\
& p(r, x)=-\frac{1}{r^{3}(1-N)\left(1+\delta^{-2}\right)} \int_{0}^{\eta}\left\{\varphi_{0}(\xi)+G\left[\varphi_{0}(\xi)\right]+G\left[\varphi_{0}(\xi)\right] G^{\prime}\left[\varphi_{0}(\xi)\right]\right. \\
& +G\left[\varphi_{0}(\xi)\right] G^{\prime 2}\left[\varphi_{0}(\xi)\right]+\frac{1}{2} G^{\prime \prime}\left[\varphi_{0}(\xi)\right] G^{2}\left[\varphi_{0}(\xi)\right]+G\left[\varphi_{0}(\xi)\right] G^{\prime 3}\left[\varphi_{0}(\xi)\right] \\
& \left.+\frac{a}{2} G^{\prime \prime}\left[\varphi_{0}(\xi)\right] G^{\prime}\left[\varphi_{0}(\xi)\right] G^{2}\left[\varphi_{0}(\xi)\right] d \xi\right\} \\
& \eta=r-\delta^{-2} x \\
& \text { where } G\left(\varphi_{0}(\eta)\right), G^{\prime}\left(\varphi_{0}(\eta)\right), G^{n}\left(\varphi_{0}(\eta)\right) \text { are defined by (53) to (55). }
\end{aligned}
$$




\section{Conclusion}

In this paper we have investigated the information on the flow phenomena e.g. the stream function, the axial velocity, the micro-polar vector and the pressure. Using the suitable change of variables which transforms the Navier Stokes equations to the ordinary differential equations(ODE), we reduced these ODE to the nonlinear functional equation. Applying the Adomian decomposition method to the nonlinear functional equation we obtain the approximated solutions of the Navier Stokes equations.

\section{References}

[1] C. Eringen, “Theory of micro-polar fluids," Journal of Mathematics and Mechanics, 16, pp.1-16. 1966.

[2] D. Srinivacharya, M. Mishra and A.R. Rao,"Peristaltic pumping of a micro-polar fluid in a tube". Acta Mechanica, 161, pp. 165-178, 2003.

[3] T. Hayat, N. Ali and Z. Abbas, "Peristaltic flow of micro-polar fluid in a channel with different wave forms," Physics letters A, 370, pp. 331-344, 2007.

[4] W. Kwang, H. Chu and J. Fang, "Peristaltic transport in a slip flow", European Physical Journal B, 16, pp. 543-547, 2000.

[5] D. Tripathi, M.K. Chaube and P.K. Gupta, "Stokes flow of micro-polar fluids by peristaltic pumping through tube with slip boundary condition," Appl. Math. Mech. -Engl. Ed., vol.32, n0.12, pp.1587-1598, 2011.

[6] D. Tripathi, P.K. Gupta and S. Das, "Influence of slip condition on peristaltic transport of a viscoelastic fluid with fractional Burgers' model”, Thermal Science ,vol. 15, n0. 2, pp.501-515, 2011.

[7] S.K. Pandey and D. Tripathi, "Peristaltic transport of a casson fluid in a finite channel: application to flows of concentrated fluids in oesophagus", International Journal of Biomathematics, 3, pp.473-491. 2000.

[8] D. Tripathi, "Numerical and analytical simulation of peristaltic flows of generalized Oldroyd-B fluids," International Journal for Numerical Methods in Fluids," DOI 10. 1002/.d.2466 .

[9] D. Tripathi, "Numerical study on peristaltic flow of generalized Burgers' fluids in uniform tubes in presence of an endoscope", International Journal for Numerical Methods in Biomedical Engineering, vol.27, n0.11, pp.1812-1828, 2011.

[10] D. Tripathi, "Peristaltic transport of fractional Maxwell fluids in uniform tubes: application of an endoscope", Computers and Mathematics with Applications,vol. 62, n0.3, pp.1116-1126, 2011. [11] D. Tripathi, S.K. Pandey and S. Das, "Peristaltic flow of viscoelastic fluid with fractional Maxwell model through a channel," Applied Mathematics and Computation, 215, pp. 3645-3654, 2010.

[12] A. N. Shapiro, M.K. Jaferin and S.L. Weinberg, "Peristaltic pumping with long wavelengths at low Reynolds number", Journal of Fluid Mechanics, 37, pp. 799-825, 1969.

[13] G. Adomian, "A review of the decomposition method and some recent results for nonlinear equations," Comput.Math.Applic, vol.21, n0.5 pp.101-127, 1991.

[14] G. Adomian, "A review of the decomposition method and some recent results for nonlinear equations," Math.Comp.Modelling, vol. 13, n0.7, pp.17-43, 1990.

[15] Y. Cherruault, "Convergence of Adomian's method", Kybernetes, vol.18, n0.2, pp.3138, 1989.

[16] J. Jin and M. Liu, "A new modification of Adomian decomposition method for solving a kind of evolution equations”, Appl. Math. Comput., 169, pp. 953962, 2005.

[17] E.Babolian and J. Biazar, "Solution of nonlinear equations by modified Adomian decomposition method", Appl. Math. Comput., 132, pp.167-172, 2002.

[18] A.M. Wazwaz, "A reliable modification of Adomian decomposition method", Appl. Math. Comp., 102, pp. 77-86, 1999. 